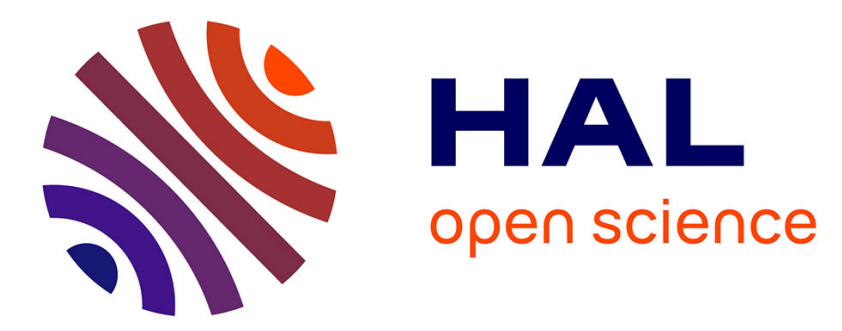

\title{
Robust Newton solver based on variable switch for a finite volume discretization of Richards equation
}

Sabrina Bassetto, Clément Cancès, Guillaume Enchéry, Quang Huy Tran

\section{To cite this version:}

Sabrina Bassetto, Clément Cancès, Guillaume Enchéry, Quang Huy Tran. Robust Newton solver based on variable switch for a finite volume discretization of Richards equation. Finite Volumes for Complex Applications IX, Jun 2020, Bergen, Norway. hal-02464945

\section{HAL Id: hal-02464945 \\ https://hal.science/hal-02464945}

Submitted on 3 Feb 2020

HAL is a multi-disciplinary open access archive for the deposit and dissemination of scientific research documents, whether they are published or not. The documents may come from teaching and research institutions in France or abroad, or from public or private research centers.
L'archive ouverte pluridisciplinaire HAL, est destinée au dépôt et à la diffusion de documents scientifiques de niveau recherche, publiés ou non, émanant des établissements d'enseignement et de recherche français ou étrangers, des laboratoires publics ou privés. 


\title{
Robust Newton solver based on variable switch for a finite volume discretization of Richards equation
}

Sabrina Bassetto, Clément Cancès, Guillaume Enchéry and Quang Huy Tran

\begin{abstract}
We propose an efficient nonlinear solver for the resolution of the Richards equation. It is based on variable switching and is easily implemented thanks to a fictitious variable allowing to describe both the saturation and the pressure. Numerical experiments show that our method enables to use Newton's method with large time steps, reasonable number of iterations and in regions where the pressure-saturation relationship is given by a graph.
\end{abstract}

Key words: Degenerate parabolic equation, nonlinear solver, variable switch

MSC (2010): 65M08, 65N08, 35Q30

\section{Finite volume approximation of the Richards equation}

The Richards equation is often used to model unsaturated flows in a porous medium $\Omega \subset \mathbb{R}^{d}(1 \leq d \leq 3)$. The fluid occupying the pore space is described by the pressure $p \in \mathbb{R}$ of the water phase and the water saturation $s \in[0,1]$, which represents the volume ratio of water in the pore space. The conservation law for the water volume then writes

$$
\partial_{t}(\phi s)-\operatorname{div}\left(\frac{\lambda}{\mu} k_{r}(s)(\nabla p-\rho \mathbf{g})\right)=0 \quad \text { in } \Omega \times \mathbb{R}_{+},
$$

where $\phi \in(0,1)$ is the porosity of $\Omega, \lambda$ its intrinsic permeability, $\mu$ the water viscosity, $\rho$ the water density and $\mathbf{g}$ the gravitational acceleration. The relative perme-

Sabrina Bassetto, Guillaume Enchéry, and Quang Huy Tran

IFPEN, $1 \&$ 4, avenue du Bois Préau, 92852 Rueil-Malmaison Cedex - France

e-mail: \{sabrina.bassetto, guillaume.enchery, quang-huy.tran\} @ifpen.fr

Clément Cancès

Inria, Univ. Lille, CNRS, UMR 8524 - Laboratoire Paul Painlevé, 59000 Lille, France

e-mail: clement.cances@inria.fr 
ability function $k_{r}:[0,1] \rightarrow \mathbb{R}^{+}$is continuous and nondecreasing, and we denote by $s_{\mathrm{rw}}=\max \left\{s \mid k_{r}(s)=0\right\}$ the residual water saturation. The saturation $s$ and pressure $p$ are linked pointwise by the relation

$$
s=\mathscr{S}(p) \quad \text { in } \Omega \times \mathbb{R}_{+},
$$

where $\mathscr{S}: \mathbb{R} \rightarrow[0,1]$ is nondecreasing and satisfies $\mathscr{S}(p)=1-s_{\mathrm{rn}}$ if $p \geq p_{b}, s_{\mathrm{rn}}$ denoting the residual saturation of air, $p_{b}$ the entry pressure and $\mathscr{S}(p) \rightarrow s_{\mathrm{rw}}$ as $p \rightarrow-\infty$. We assume that $\mathscr{S}$ is $C^{1}$ and convex on $\left(-\infty, p_{\mathrm{s}}\right)$, and $C^{1}$ and concave on $\left(p_{\mathrm{s}},+\infty\right)$ for some $p_{\mathrm{s}} \leq 0$. We denote by $s_{\mathrm{s}}=\mathscr{S}\left(p_{\mathrm{s}}\right)$. The above assumptions on $k_{r}$ and $\mathscr{S}$ are satisfied by the classical Brooks-Corey and van Genuchten-Mualem models respectively given by

$$
\begin{aligned}
& k_{r \mathrm{BC}}(s)=s_{\mathrm{eff}}^{3+\frac{2}{n}}, \quad \mathscr{S}_{\mathrm{BC}}(p)= \begin{cases}s_{\mathrm{rw}}+\left(1-s_{\mathrm{rn}}-s_{\mathrm{rW}}\right)\left(\frac{p}{p_{b}}\right)^{-n} & \text { if } p \leq p_{b}, \\
1-s_{\mathrm{rn}} & \text { if } p>p_{b},\end{cases} \\
& k_{r \mathrm{VGM}}(s)=s_{\mathrm{eff}}^{\frac{1}{2}}\left\{1-\left[1-s_{\mathrm{eff}}^{\frac{1}{m}}\right]^{m}\right\}^{2}, \quad \mathscr{S}_{\mathrm{vGM}}(p)= \begin{cases}s_{\mathrm{rW}}+\frac{1-s_{\mathrm{r}}-s_{\mathrm{rW}}}{\left[1+\left|\frac{\alpha}{\rho g} p\right|^{n}\right]^{m}} & \text { if } p \leq p_{b} \\
1-s_{\mathrm{rn}} & \text { if } p>p_{b}\end{cases}
\end{aligned}
$$

where $s_{\mathrm{eff}}=\frac{s-s_{\mathrm{rw}}}{1-s_{\mathrm{r}}-s_{\mathrm{rw}}}$ and, for the van Genuchten-Mualem model, $m=1-\frac{1}{n}$ and $p_{b}=0 P a$. Dirichlet boundary conditions are imposed on a part $\Gamma^{D}$ of $\partial \Omega$, while inflow Neumann boundary conditions are imposed on the complement $\Gamma^{N}=\partial \Omega \backslash$ $\Gamma^{D}$ :

$$
p=p_{D} \text { on } \Gamma^{D} \times \mathbb{R}_{+}, \quad-\frac{\lambda}{\mu} k_{r}(s)(\nabla p-\rho \mathbf{g}) \cdot \mathbf{n}=q_{N} \quad \text { on } \Gamma^{N} \times \mathbb{R}_{+},
$$

with $q_{N} \leq 0$. Finally, the system is closed by prescribing an initial saturation profile

$$
s(\cdot, 0)=s^{0} \quad \text { in } \Omega, \quad \text { with } \quad 0 \leq s^{0} \leq 1 .
$$

We refer to [2] for further details on the modeling and to [1] for the well-posedness of the problem.

The problem (1), (2), (5), and (6) is discretized by means of a finite-volume scheme: an upstream mobility is used for convection and a two-point flux approximation (TPFA) for the capillary diffusion. Let $\left(\mathscr{T}, \mathscr{E},\left(\mathbf{x}_{K}\right)_{K \in \mathscr{T}}\right)$ be a finite volume mesh of $\Omega$ fulfilling the classical orthogonality condition required for the consistency of TPFA. Since this notion is classical, we remain sloppy here on the definition and refer to [6, Definition 9.1] for details. Let us just mention that $\mathscr{T}$ denotes the set of the cells, while the set of the edges $\mathscr{E}$ is partitioned into the set of the internal edges $\mathscr{E}_{\text {int }}=\{\sigma \in \mathscr{E}|\sigma=K| L=\partial K \cap \partial L\}$, the set of the Dirichlet boundary edges $\mathscr{E}_{\text {ext }}^{D}=\left\{\sigma \in \mathscr{E} \mid \sigma \subset \Gamma^{D}\right\}$, and the set of the Neumann boundary edges $\mathscr{E}_{\mathrm{ext}}^{N}=\{\sigma \in$ $\left.\mathscr{E} \mid \sigma \subset \Gamma^{N}\right\}$. We denote by $\mathscr{E}_{K}=\{\sigma \in \mathscr{E} \mid \sigma \subset \partial K\}$. For the time discretization, we allow for non-uniform time steps $\tau_{n}=t^{n}-t^{n-1}, n \geq 1$. At initial time $t=0, s^{0}$ is 
discretized into $s_{K}^{0}=\frac{1}{|K|} \int_{K} s^{0}$. For $\sigma \in \mathscr{E}_{\text {int }} \cup \mathscr{E}_{\text {ext }}^{D}, \sigma \in \mathscr{E}_{K}$, we define the mirror value $u_{K, \sigma}^{n}$ of $u_{K}^{n}$ across $\sigma$ by $u_{K, \sigma}^{n}=u_{L}^{n}$ if $\sigma=K \mid L \in \mathscr{E}_{\text {int }}$ and $u_{K, \sigma}^{n}=u_{\sigma}^{n}=\frac{1}{\tau_{n}|\sigma|} \int_{\sigma} \int_{t^{n-1}}^{t^{n}} u^{D}$ if $\sigma \in \mathscr{E}_{\mathrm{ext}}$. The conservation of the water phase is discretized into

$$
\phi_{K} \frac{s_{K}^{n}-s_{K}^{n-1}}{\tau_{n}}|K|+\sum_{\sigma \in \mathscr{E}_{K}} F_{K \sigma}^{n}=0, \quad K \in \mathscr{T}, n \geq 1 .
$$

The expression of the fluxes relies on a unique upwinding for capillary diffusion and for gravitationally induced convection, that is

$$
F_{K \sigma}^{n}= \begin{cases}A_{\sigma}\left\{\frac{k_{r \sigma, u p}^{n}}{\mu}\left[\left(p_{K}^{n}-p_{K, \sigma}^{n}\right)+\rho g\left(z_{K}-z_{K, \sigma}\right)\right]\right\} & \text { if } \sigma \in \mathscr{E}_{\mathrm{int}} \cup \mathscr{E}_{\mathrm{ext}}^{D}, \\ \frac{1}{\tau_{n}} \int_{t^{n-1}}^{t^{n}} \int_{\sigma} q_{N} & \text { if } \sigma \in \mathscr{E}_{\mathrm{ext}},\end{cases}
$$

where

$$
\begin{aligned}
k_{r \sigma, u p}^{n} & = \begin{cases}k_{r}\left(s_{K}^{n}\right) & \text { for }\left(p_{K}^{n}-p_{K, \sigma}^{n}\right)+\rho g\left(z_{K}-z_{K, \sigma}\right) \geq 0, \\
k_{r}\left(s_{K, \sigma}^{n}\right) & \text { otherwise, }\end{cases} \\
A_{\sigma} & = \begin{cases}m_{\sigma} \frac{\lambda_{K} \lambda_{L}}{\lambda_{L} d_{K, \sigma}+\lambda_{K} d_{L, \sigma}} & \text { if } \sigma=K \cap L, \\
m_{\sigma} \frac{\lambda_{K}}{d_{K, \sigma}} & \text { if } \sigma \in \mathscr{E}_{\mathrm{ext}}^{D},\end{cases}
\end{aligned}
$$

with $\lambda_{K}=\lambda\left(\mathbf{x}_{K}\right), d_{K, \sigma}=\left|\mathbf{x}_{K}-\mathbf{x}_{L}\right|$ if $\sigma=K \mid L \in \mathscr{E}_{\text {int }}, d_{K, \sigma}=\operatorname{dist}\left(\mathbf{x}_{K}, \sigma\right)$ if $\sigma \in \mathscr{E}_{\mathrm{ext}}^{D}$ and $m_{\sigma}$ is the Lebesgue measure of the edge $\sigma$. The discrete water saturation and pressure are related cellwise by the relation

$$
s_{K}^{n}=\mathscr{S}\left(p_{K}^{n}\right), \quad K \in \mathscr{T}, n \geq 1 .
$$

The scheme (7)-(11) admits a unique discrete solution $\left(s_{K}^{n}, p_{K}^{n}\right)_{K \in \mathscr{T}}$ for all $n \geq 1$ and converges as the mesh size and the time step tend to 0 (this will be proved in a forthcoming work). In this contribution, we rather focus on the practical resolution of the nonlinear system (7)-(11) via an iterative method. For our works, we choose to use Newton's method. Notice that the physical models presented above, both feature two difficulties for Newton's method: the function $\mathscr{S}_{\mathrm{BC}}$ is Lipschitz continuous but not $C^{1}$ and the mobility function $k_{r \mathrm{VGM}}$ is singular at $s=1-s_{\mathrm{rn}}$ where the derivative blows up.

\section{Fictitious variable and Newton's method}

A natural approach to solve the nonlinear system (7)-(11) is to choose $\left(p_{K}\right)_{K \in \mathscr{T}}$ as a primary unknown and to solve the corresponding nonlinear system thanks to Newton's method (or alternatively some modified Picard's method, see e.g. [9]). However, the choice of the pressure as the primary variable is known to be inefficient for dry soils $s \ll 1$ where they are outperformed by schemes using $s$ as primary variable. On the other hand, the knowledge of the saturation is not sufficient to describe the pressure in saturated regions where $s=1$. This motivated the introduction 
of schemes based on variable switching between $s$ and $p$, see [7, 5]. Our approach is based on [3] and can be seen as a reformulation of the variable switch which makes its implementation much easier. Unlike in [3], we do not use the Kirchhoff transform which cannot be easily computed for the van Genuchten-Mualem model. The idea is to choose a parametrization of the graph $\{p, \mathscr{S}(p)\}$, i.e. to choose two functions $\mathfrak{s}: I \rightarrow\left[s_{\mathrm{rw}}, 1-s_{\mathrm{rn}}\right]$ and $\mathfrak{p}: I \rightarrow \mathbb{R}$ such that $\mathfrak{s}(u)=\mathscr{S}(\mathfrak{p}(u))$ for all $u \in I \subset \mathbb{R}$. Such a parametrization is not unique: one can for instance choose $I=\mathbb{R}, \mathfrak{p}=\operatorname{Id}$ and $\mathfrak{s}=\mathscr{S}$, or $\mathfrak{p}=(\operatorname{Id}+\mathscr{S})^{-1}$ and $\mathfrak{s}=\left(\operatorname{Id}+\mathscr{S}^{-1}\right)^{-1}$ so that $\mathfrak{s}^{\prime}(u)+\mathfrak{p}^{\prime}(u)=1$ for all $u \in \mathbb{R}$. Here, we rather set $I=\left(s_{\mathrm{rw}},+\infty\right)$ and

$$
\mathfrak{s}(u)=\left\{\begin{array}{ll}
u & \text { if } u \leq u_{\mathrm{s}}, \\
\mathscr{S}\left(p_{\mathrm{s}}+\frac{u-u_{\mathrm{s}}}{\mathscr{S}^{\prime}\left(p_{\mathrm{s}}^{-}\right)}\right) & \text {if } u \geq u_{\mathrm{s}},
\end{array} \quad \mathfrak{p}(u)= \begin{cases}\mathscr{S}^{-1}(u) & \text { if } u \leq u_{\mathrm{s}}, \\
p_{\mathrm{s}}+\frac{u-u_{\mathrm{s}}}{\mathscr{S}^{\prime}\left(p_{\mathrm{s}}^{-}\right)} & \text {if } u \geq u_{\mathrm{s}} .\end{cases}\right.
$$

where $\mathscr{S}^{\prime}\left(p_{\mathrm{s}}^{-}\right)$denotes the limit of $\mathscr{S}^{\prime}(p)$ as $p$ tends to $p_{\mathrm{s}}$ from below. Since $\left(p_{\mathrm{s}}, u_{\mathrm{s}}\right)$ is the inflexion point of $\mathscr{S}$, both $\mathfrak{s}$ and $\mathfrak{p}$ are $C^{1}$ and concave, and even $C^{2}$ if $\mathscr{S}$ is given by (4). Moreover, for all $p \in \mathbb{R}$, there exists a unique $u \in\left(s_{\mathrm{rw}},+\infty\right)$ such that $(p, \mathscr{S}(p))=(\mathfrak{p}(u), \mathfrak{s}(u))$.

Choosing $u$ as a primary variable in the scheme (7)-(11) amounts to search for $\mathbf{u}^{n}=$ $\left(u_{K}^{n}\right)_{K \in \mathscr{T}}$ such that $s_{K}^{n}=\mathfrak{s}\left(u_{K}^{n}\right)$ and $p_{K}^{n}=\mathfrak{p}\left(u_{K}^{n}\right)$ for all $K \in \mathscr{T}$. Equation (11) is then automatically satisfied. The resulting system $\mathscr{F}_{n}\left(\mathbf{u}^{n}\right)=\mathbf{0}$ made of $N_{\mathscr{T}}=\operatorname{Card}(\mathscr{T})$ nonlinear equations admits a unique solution $\mathbf{u}^{n}$ since it is fully equivalent to (7)(11). However, the nonlinear change of variable to pass from $\mathbf{p}^{n}=\left(p_{K}^{n}\right)_{K \in \mathscr{T}}$ to $\mathbf{u}^{n}$ as primary variable strongly impacts the nonlinear solver. Our approach is based on Newton's method, that is detailed in Algorithm 1 and that include the following procedures in order to handle difficulties which are inherent to the chosen petrophysical models.

- $\operatorname{check}()$ and update ()

The law of the relative permeability $k_{r}$, in the van Genuchten-Mualem case (4), has very large derivative values, which can be equal to $\infty$ for $s \rightarrow 1$. In order to overcome this difficulty, we approximate $k_{r \mathrm{vGM}}$, during Newton's iterations, for $s \in N=\left[s_{\text {lim }}, 1\right]$, with a polynomial $\tilde{k}_{r \mathrm{VGM}}(s)$ of second degree which satisfies the following conditions: $k_{r}\left(s_{\lim }\right)=\tilde{k}_{r \mathrm{VGM}}\left(s_{\lim }\right), k_{r}^{\prime}\left(s_{\lim }\right)=\tilde{k}_{r_{\mathrm{VGM}}}^{\prime}\left(s_{\lim }\right), k_{r}^{\prime \prime}\left(s_{\mathrm{lim}}\right)=$ $\tilde{k}_{r_{\mathrm{VGM}}}^{\prime \prime}\left(s_{\mathrm{lim}}\right)$. The idea is to progressively increase the value of $s_{\mathrm{lim}}$ in order to recover the real law at convergence. The function $\operatorname{check}()$ verifies the error we commit in the approximation. If this error is smaller than a fixed tolerance, namely $\left|k_{r_{\mathrm{VGM}}}(1)-\tilde{k}_{r_{\mathrm{VGM}}}(1)\right|<\varepsilon_{k_{r}^{\mathrm{VGM}}}$, it returns true, false otherwise. At each Newton's iteration, we increase the value of $s_{\text {lim }}$ thanks to the function update(). The increment speed depends on the norm of the residual. Let us call $\delta_{s_{w, \max }}=1-s_{\mathrm{rn}}-s_{\mathrm{lim}}$. If $\left\|\mathscr{F}_{n}\left(\mathbf{u}^{n, i}\right)\right\|_{\infty}>\mathcal{E}_{\mathscr{F}_{\mathrm{vGM}}}$ we set $\delta_{s_{w, \text { max }}}=\delta_{S_{w, \text { max }}} \cdot \omega$ and $\delta_{s_{w, \text { max }}}=\delta_{s_{w, \text { max }}}^{2}$ otherwise, with $\omega<1$.

- $\operatorname{truncation}()$

Since $\mathscr{F}_{n}$ is not necessarily $C^{1}$ ( $\mathscr{S}_{\mathrm{BC}}$ is not $C^{1}$ in the Brooks and Corey case), 
following $[8,10]$, the Newton increment is truncated near the inflection point $s_{\mathrm{s}}$, as described in Algorithm 2.

- decreaseDeltaTime() and increaseDeltaTime()

In our numerical tests, we increase the time step in such a way that $\Delta t^{n+1}=$ $\min \left(\Delta t_{\max }, \alpha_{\Delta t}^{+} \cdot \Delta t^{n}\right)$ and decrease it in such a way that $\Delta t^{n+1}=\max \left(\alpha_{\Delta t}^{-}\right.$. $\left.\Delta t^{n}, \Delta t_{\min }\right)$ with $\alpha_{\Delta t}^{+}>1$ and $\alpha_{\Delta t}^{-}<1$. If $\Delta t_{\min }$ is reached, the simulation stops.



\section{Numerical results}

For the numerical validation of our scheme, we consider two tests inspired from those proposed in [4]. These two tests make use of the classical Brooks and Corey and Van Genuchten-Mualem models. For the simulations we take the following parameters: $\varepsilon=10^{-12}, i_{\max }=500, \varepsilon_{k_{r}^{\mathrm{VGM}}}=10^{-3}, \varepsilon_{\mathscr{F}_{\mathrm{vGM}}}=10^{-9}, \varepsilon_{\delta}=10^{-6}, \alpha_{\Delta t}^{-}=$ $0.5, \omega=0.07$. As in [4], our aim is here just to improve the robustness of the Newton's algorithm when used with the TPFA scheme. Therefore, our study here focuses on the corresponding nonlinear system even if more accurate schemes could be used to better take into account the heterogeneities, in particular the ones related to the capillary pressures.

\section{Test 1 with the Brooks and Corey model}

In this test we simulate a vertical drainage through a layered soil $\Omega=[0 \mathrm{~m}, 2 \mathrm{~m}]$ from initially saturated conditions during a time interval $[0, T]$ with $T=105 \cdot 10^{4} \mathrm{~s}$. At the initial time the pressure varies with respect to the height of the column, that is $p^{0}(z)=-\rho g(z-2)$, where $\rho=10^{3} \mathrm{~kg} \cdot \mathrm{m}^{-3}$ and $g=9.81 \mathrm{~m} \cdot \mathrm{s}^{-2}$. During the 
simulation, we impose a Dirichlet boundary condition $p_{D}=0 P a$ on the bottom of the column and a no-flow boundary condition on the top. The soil is made of two rock types: RT1 for $0 m<z<0.6 m$ and $1.2 m<z<2 m$, and RT2 for $0.6 m<z<$ $1.2 \mathrm{~m}$. Their hydraulic properties are given in Table 1. Simulations are performed on a mesh with 1000 cells and an initial time step $\Delta t_{i n i}=2000 \mathrm{~s}$ which increases after the first time iteration up to $\Delta t_{\max }=2 \cdot \Delta t_{i n i}$ using $\alpha_{\Delta t}^{+}=1.2$. The truncation procedure, detailed in Algorithm 2, is activated during Newton's iterations.

Table 1: Hydraulic properties for Test 1

\begin{tabular}{|c|c|c|c|c|c|c|}
\hline & $1-s_{\mathrm{rn}}$ & $s_{\mathrm{rw}}$ & $p_{b}[\mathrm{~Pa}]$ & $n$ & $\lambda\left[m^{2}\right]$ & $\phi$ \\
\hline RT1 & 1.0 & 0.2 & $-3.4301 \cdot 10^{3}$ & 1.5 & $10^{-13}$ & 0.35 \\
\hline RT2 & 1.0 & 0.1 & $-1.4708 \cdot 10^{3}$ & 3.0 & $10^{-11}$ & 0.35 \\
\hline
\end{tabular}

Table 2 gives the average number of iterations of the nonlinear solvers used here and in [4] along with the number of time steps.

Table 2: Performances of the nonlinear (nl) solvers for Test 1

\begin{tabular}{lll}
\hline & $\sharp$ total nl iterations & $\sharp$ time iterations \\
\hline Our method & 1118 & 265 \\
Method proposed in [4] (coarser mesh) & 4469 (inner iterations) & 300 \\
\hline
\end{tabular}

Note that a coarser mesh has been used in [4] for this test. Solutions obtained at the final time are shown in Figure 1. In some areas, pressures are higher than the entry pressure and the saturation-pressure relationship is there no more a function. The problem can still be solved thanks to the use of the parametrization technique. Figure 2 shows the evolution of the average Newton's convergence rate given, for a time step $n$, by $\left.C V_{\text {rate }}^{n}=\frac{1}{N_{\text {iter }}^{n}} \sum_{i=1}^{N_{\text {iter }}^{n}} \frac{\log _{10}\left\|\mathscr{F}_{n}\left(\mathbf{u}^{n, i}\right)\right\|_{\infty}}{\log _{10} \| \mathscr{F}_{n}\left(\mathbf{u}^{n}, i-1\right.}\right) \|_{\infty}$. The rate is on the whole equal to 2. Negative rates are due to residual norms which are greater than one at some iterations.



Fig. 1: Evolution in time of the saturation on the left and of the pressure on the right 


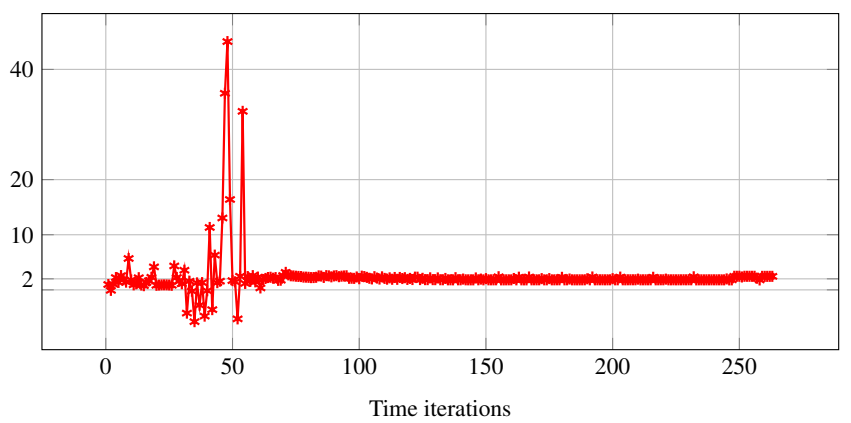

Fig. 2: Test 1: Evolution of the average Newton's convergence rate during time iterations.

\section{Test 2 with the Van Genuchten-Mualem model}

In this test, starting from an initially very dry layered domain, $\Omega=[0 \mathrm{~m}, 1 \mathrm{~m}] \times$ $[-3 m, 0 m]$, made of sand and clay, water flows from the top of the structure as shown in Figure 3. The hydraulic properties of the rock types are given in Table 3. The initial pressure is set to $-47.088 \cdot 10^{5} \mathrm{~Pa}$. A no-flow boundary condition is applied everywhere except on the top sand surface where the water flux rate is equal to $0.5 \mathrm{~m} /$ day. The simulation is performed on a mesh composed of a $100 \times 60$ cells during a time interval $[0, T]$ with $T$ equal to one day. We use an initial time step $\Delta t_{\text {ini }}=25 \cdot 10^{2} \mathrm{~s}$ which increases after the first time iteration up to $\Delta t_{\max }=3 \cdot \Delta t_{\text {ini }}$ using $\alpha_{\Delta t}^{+}=2$.

Table 3: Hydraulic properties for Test 2

\begin{tabular}{lll}
\hline & RT1 (Sand) & RT2 (Clay) \\
\hline $1-s_{\mathrm{rn}}$ & 1.0 & 1.0 \\
$s_{\mathrm{rw}}$ & 0.0782 & 0.2262 \\
$n$ & 2.239 & 1.3954 \\
$\lambda\left[\mathrm{m}^{2}\right]$ & $6.3812 \cdot 10^{-12}$ & $1.5461 \cdot 10^{-13}$ \\
$\alpha\left[\mathrm{m}^{-1}\right]$ & 2.8 & 1.04 \\
$s_{\lim }$ & 0.985 & 0.985 \\
$\phi$ & 0.3658 & 0.4686 \\
\hline
\end{tabular}



Fig. 3: Configuration of the domain for Test 2.

During this simulation, the relative permeability is approximated following the strategy which has been previously described and activating the $\operatorname{check}()$ and update () procedures. The truncation method is not required here because $\mathscr{S}_{\mathrm{vGM}}$ is $C^{2}$. Table 4 gives the average number of iterations of the nonlinear solvers used here and in [4] along with the number of time steps. Solutions obtained at the final time are shown in Figure 4. Figure 5 shows the evolution of the average Newton's convergence rate which is slightly bigger than 1 . The loss of the quadratic convergence may be due to the low regularity of the laws and to the use of the approximation $\tilde{k}_{r v G M}$. 
Table 4: Performances of the nonlinear (nl) solvers for Test 2

\begin{tabular}{lll}
\hline & $\sharp$ total nl iterations & $\sharp$ time iterations \\
\hline Our method & 151 & 13 \\
Method proposed in [4] & 482 (inner iterations) & 24 \\
\hline
\end{tabular}
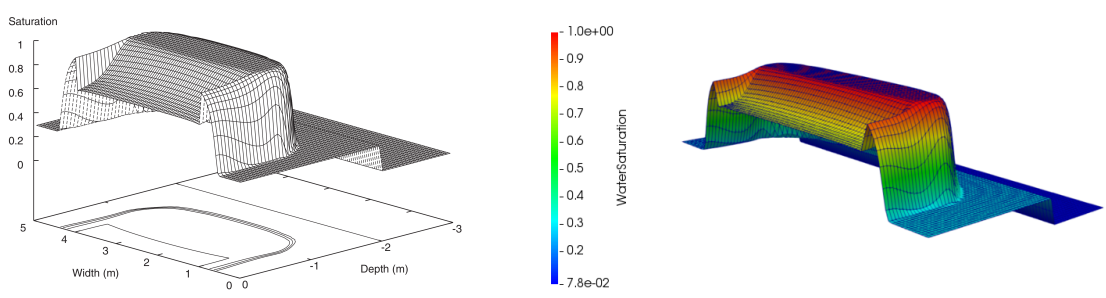

Fig. 4: At the final time for Test 2: $s$ obtained in [4] (left) and with our solution (right).



Fig. 5: Test 2: Evolution of the average Newton's convergence rate during time iterations.

\section{References}

1. Alt, H.W., Luckhaus, S.: Quasilinear elliptic-parabolic differential equations. Math. Z. 183(3), 311-341 (1983)

2. Bear, J., Bachmat, Y.: Introduction to modeling of transport phenomena in porous media. Kluwer Academic Publishers, Dordrecht, The Netherlands (1990)

3. Brenner, K., Cancès, C.: Improving Newton's method performance by parametrization: The case of the Richards equation. SIAM J. Numer. Anal. 55(4), 1760-1785 (2017)

4. Casulli, V., Zanolli, P.: A nested Newton-type algorithm for finite volume methods solving Richards' equation in mixed form. SIAM J. Sci. Comp. 32(4), 2255-2273 (2010)

5. Diersch, H.J.G., Perrochet, P.: On the primary variable switching technique for simulating unsaturated-saturated flows. Adv. Water Resour. 23(3), 271-301 (1999)

6. Eymard, R., Gallouët, T., Herbin, R.: Finite volume methods. Ciarlet, P. G. (ed.) et al., in Handbook of numerical analysis. North-Holland, Amsterdam, pp. 713-1020 (2000)

7. Forsyth, P.A., Wu, Y.S., Pruess, K.: Robust numerical methods for saturated-unsaturated flow with dry initial conditions in heterogeneous media. Adv. Water Resour. 18, 25-38 (1995)

8. Jenny, P., Tchelepi, H.A., Lee, S.H.: Unconditionally convergent nonlinear solver for hyperbolic conservation laws with S-shaped flux functions. J. Comput. Phys. 228(20), 7497-7512 (2009)

9. List, F., Radu, F.A.: A study on iterative methods for solving Richards equation. Comput. Geosci. 20(2), 341-353 (2016)

10. Wang, X., Tchelepi, H.A.: Trust-region based solver for nonlinear transport in heterogeneous porous media. J. Comput. Phys. 253, 114-137 (2013) 